\title{
Editorial
}

\section{Cold plasmas: fundamentals and applications}

\author{
Gianpiero Colonna $^{\mathrm{a}}$, Mario Capitelli ${ }^{\mathrm{b}}$, and Annarita Laricchiuta ${ }^{\mathrm{c}}$ \\ CNR Institute for Plasma Science and Technology (ISTP) Bari Section, Via Amendola 122/D, Bari, Italy \\ Published online 13 September 2021 \\ (C) The Author(s), under exclusive licence to EDP Sciences, SIF and Springer-Verlag GmbH Germany, \\ part of Springer Nature 2021
}

\begin{abstract}
The Editorial of the Topical Issue Cold Plasmas: Fundamentals and Applications provides the general introduction to the collection of Topical Reviews, offering a reading key for the motivation and the design of the issue, illustrating the paradigm in the study of low-temperature plasmas through selected applications of large interest and of significant technological impact.
\end{abstract}

This Topical Issue is devoted to cold plasmas and presents the modern approaches for the theoretical and experimental investigation, moving from principles to applications. The Topical Issue stems from the $63 \mathrm{rd}$ Course "Cold Plasmas: Fundamentals and Applications" of the International School of Quantum Electronics, held on September 2019 in the Ettore Majorana Center for Scientific Culture in Erice (Italy) [1], that had, among the lecturers, internationally acknowledged experts of the plasma scientific community, active in different fields.

The Topical Issue aims at giving a thorough and up-to-date overview of the research in the field, covering different aspects of the low-temperature plasmas, including the theoretical description, from elementary processes to the fundamentals of modeling, the diagnostics techniques for plasma characterization, and the actual technological exploitation.

The non-equilibrium in the internal levels and in the energy distribution of free electrons (eedf) drives the plasma evolution, activating mechanisms that can efficiently lead to the desired specific reaction products and in this Topical Issue the assessed method in kinetic modeling, namely the state-to-state approach, implementing complex chemical-physics models detailed on the quantum state of chemical species, is presented. Excited states are key in the plasma chemistry and the predictive character of simulation codes is strictly linked to the accurate and detailed description of the collisional dynamics and to the knowledge of cross sections and rate coefficients for elementary processes in the plasma phase and on the surface [2]. Diagnostics serves for the experimental investigation on plasma properties and spectroscopic techniques have been exploited also for the derivation of temperatures

\footnotetext{
${ }^{\mathrm{a}}$ e-mail: gianpiero.colonna@cnr.it

b e-mail: mario.capitelli@ba.istp.cnr.it

c e-mail: annarita.laricchiuta@cnr.it (corresponding author)
}

characterizing internal distributions, thus allowing in specific cases the model validation.

The Topical Issue collects five Topical Reviews (TR) focused on topics that have attracted large interest in the community due to their potential impact in different fields, ranging from energy and material processing to environment and agriculture, demonstrating the relevance of plasmas in future enabling technologies. The topics comprise:

- discharges for material processing, sterilization, synthesis of chemicals

- plasma-assisted combustion for new-concept highefficiency engines

- negative ion sources for neutral beam injection in fusion reactors

- $\mathrm{CO}_{2}$ plasma activation for environment-friendly energy production

- plasma-assisted agriculture for pre-harvest and post-harvest processing.

In the TR about discharges [3], the fundamentals of the modeling of low-temperature plasmas are discussed, presenting a state-to-state kinetic model selfconsistently coupled with the eedf, emphasizing the relevance of superelastic collisions, the concept of temperature for vibrational non-equilibrium, and illustrating a drift-diffusion model for high pressure dielectric barrier discharges. Two low-temperature plasma applications aimed at sterilization (a DBD for the efficient generation of oxidizers) and material deposition (the growth of synthetic diamonds for jewelry) are also presented. Additionally, a section is devoted to high-temperature plasmas, discussing the role of collisions in collisionless plasmas and one application relevant to the ammonia formation as an undesirable side effect in nitrogen seeding for the power exhaust in fusion devices.

The plasma-assisted combustion is a promising technology to improve the efficiency of engines, influencing 
the complex combustion chemistry, and in turn the ignition delay, the flame stability and velocity and the burning properties. In the TR [4] the combustion-related kinetics is comprehensively described, enlightening the mechanisms activated in the collisional dynamics by excited states, either electronically or ro-vibrationally, and radicals with enhancement of reaction rates, showing the relevance of a state-to-state approach in modeling. Experiments, in different type of discharges (nanosecond $\mathrm{DBD}$ and surface $\mathrm{DBD}$, repetitively nanosecond pulse discharges NRPD), are presented to demonstrate how plasma can assist the ignition, in ordinary and detonation regimes, and the combustion.

In the thematic of energy, the huge efforts of the fusion community in the construction of ITER, led to a number of side technological problems, such as the optimization of efficiency in the generation of negative ions for neutral beam injection. The TR [5] illustrates the wide spectrum of technological solution towards Cs-free sources, i.e., sources where the $\mathrm{H}^{-}$yield is controlled by volume and surface processes leading to the maximum efficiency for the dissociative attachment, including the investigation on ceramic materials characterized by low work-functions for negative ion surface production. The negative ion sources in the European labs (RAID, PROMETHEUS-I, NIO1, ELISE ...) based on different plasma generation (helicon antenna, electron cyclotron resonance, radiofrequency in ICP regime) are presented. The theoretical tools for the numerical modeling are discussed from the early global kinetic models applied to multicusp sources to Particle-In-Cell models with Monte-Carlo-Collisions used to simulate the plasma properties in the expansion chamber of an ITER-like negative ion source.

$\mathrm{CO}_{2}$ activation via plasma has gained large interest in the community. The research activity aimed at demonstrating the feasibility and controlling the efficiency in the reduction of $\mathrm{CO}_{2}$ emission in the atmosphere, for the conversion of existing energy and transport infrastructures in a perspective of environment sustainability. Besides and more fundamentally, the strategy in the investigation of this system could set a new paradigm for complex plasmas of triatomic species characterized by the non-equilibrium of vibrational modes. The knowledge gathered on $\mathrm{CO}_{2}$ plasmas is thoroughly presented in the TR [6], focusing on $0 \mathrm{D}$ global models (including detailed vibrational and excited-state kinetics either self-consistently coupled or uncoupled to eedf), on the design of experiments that provide information on the vibrational temperature (infrared spectroscopy, Raman scattering) and on the reactivity of transient species (Flow Tube experiments), considering different type of discharges (DBD, glow discharges, microwave, nano-pulsed) and hypersonic flows for aerospace applications. Moreover, fluidmodels for real geometry simulations, implementing reduced kinetic schemes, are presented.

The possibility of exposing living tissues to cold plasmas has been demonstrated, triggering the development of plasma medicine for a number of different applications, from wound healing to cancer treatment [7]. How- ever, the exploitation of plasma technologies in agriculture is a promising emerging field, being the plasma effective in different stages of the agricultural production chain, from the control of pathogens to the seed conditioning and plant growth enhancement by irrigation with plasma-activated water (PAW). The TR [8] discusses the main mechanisms of plasma interaction with seeds and plants, identifying the active agents (namely reactive oxygen, ROS, and nitrogen species, RNS, radiation, high-energy electrons, ...), and illustrates the typology, configuration and regimes of discharges used in plasma-assisted agriculture in direct, indirect and remote treatments.

It is worth noticing that, despite our efforts, some relevant topics such as nano-plasmas [9], dusty plasmas [10] and plasma generated in laser-matter interaction [11], have not been included.

The Topical Issue provides a state-of-the-art of the most recent advancements in the selected topics, illustrating the general approach in the theoretical/experimental investigation of non-equilibrium plasma systems, giving a detailed picture of the key-role played by excited species in the driving mechanisms, highlighting the numerous advantages connected to the energy efficient and environment-respectful character of the plasma technologies and also identifying the future challenges the next generation of cold plasma scientists will have to face.

We would like to warmly thank all the authors for their invaluable contribution to the Topical Issue, especially for the extra-efforts needed during this difficult time of pandemic. We extend our thanks also to the lecturers and the students of the Course [1], the former for their interesting and attractive presentations and the latter for having been the young engine of the Course. Finally, we want to express our gratitude to the International School of Quantum Electronics and to the Ettore Majorana Center for Scientific Culture in Erice, for giving us the chance of organizing the Course in a very nice and inspiring environment.

Data Availability Statement This manuscript has no associated data or the data will not be deposited. [Authors' comment: No data have been generated or are associated to the Editorial.]

\section{References}

1. 63rd Course "Cold Plasmas: Fundamentals and Applications" of the International School of Quantum Electronics, September 8-14, 2019 (2019). https://users.ba. cnr.it/istp/istpal18/ColdPlasmas/

2. M. Capitelli, R. Celiberto, G. Colonna, F. Esposito, C. Gorse, K. Hassouni, A. Laricchiuta, S. Longo, Fundamental Aspects of Plasma Chemical Physics: Kinetics, Springer Series on Atomic, Optical, and Plasma Physics, vol. 85. (Springer, Berlin, 2016)

3. G. Colonna, C.D. Pintassilgo, F. Pegoraro, A. Cristofolini, A. Popoli, G. Neretti, A. Gicquel, O. Duigou, 
T. Bieber, K. Hassouni et al., Eur. Phys. J. D 75, 183 (2021)

4. S. Starikovskaia, D.A. Lacoste, G. Colonna, Eur. Phys. J. D 75, 231 (2021)

5. F. Taccogna, S. Bechu, A. Aanesland, P. Agostinetti, R. Agnello, S. Aleiferis, T. Angot, V. Antoni, M. Bacal, M. Barbisan et al., Eur. Phys. J. D 75, 227 (2021)

6. L.D. Pietanza, O. Guaitella, V. Aquilanti, A. Armenise, I. Bogaerts, M. Capitelli, G. Colonna, V. Guerra, R. Engeln, E. Kustova, A. Lombardi, Eur. Phys. J. D 75, 237 (2021)
7. A.A. Fridman, G.G. Friedman, Plasma Medicine (Wiley, Chichester, 2013)

8. M. Šimek, T. Homola, Eur. Phys. J. D 75, 210 (2021)

9. K.K. Ostrikov, Plasma Phys. Control. Fusion 61, 014028 (2018)

10. V.E. Fortov, A.G. Khrapak, S.A. Khrapak, V.I. Molotkov, O.F. Petrov, Phys. Uspekhi 47, 447 (2004)

11. M. Capitelli, A. Casavola, G. Colonna, A. De Giacomo, Spectrochim. Acta Part B At. Spectrosc. 59, 271 (2004) 